\title{
Whole-genome sequencing of Aspergillus tubingensis G131 and overview of its secondary metabolism potential
}

\author{
Elodie Choque ${ }^{1,2}$, Christophe Klopp ${ }^{3}$, Sophie Valiere ${ }^{4}$, José Raynal ${ }^{1}$ and Florence Mathieu ${ }^{1 *}$
}

\begin{abstract}
Background: Black Aspergilli represent one of the most important fungal resources of primary and secondary metabolites for biotechnological industry. Having several black Aspergilli sequenced genomes should allow targeting the production of certain metabolites with bioactive properties.

Results: In this study, we report the draft genome of a black Aspergilli, A. tubingensis G131, isolated from a French Mediterranean vineyard. This $35 \mathrm{Mb}$ genome includes 10,994 predicted genes. A genomic-based discovery identifies 80 secondary metabolites biosynthetic gene clusters. Genomic sequences of these clusters were blasted on 3 chosen black Aspergilli genomes: A. tubingensis CBS 134.48, A. niger CBS 513.88 and A. kawachii IFO 4308. This comparison highlights different levels of clusters conservation between the four strains. It also allows identifying seven unique clusters in A. tubingensis G131. Moreover, the putative secondary metabolites clusters for asperazine and naphthogamma-pyrones production were proposed based on this genomic analysis. Key biosynthetic genes required for the production of 2 mycotoxins, ochratoxin A and fumonisin, are absent from this draft genome. Even if intergenic sequences of these mycotoxins biosynthetic pathways are present, this could not lead to the production of those mycotoxins by A. tubingensis G131.
\end{abstract}

Conclusions: Functional and bioinformatics analyses of A. tubingensis G131 genome highlight its potential for metabolites production in particular for TAN-1612, asperazine and naphtho-gamma-pyrones presenting antioxidant, anticancer or antibiotic properties.

Keywords: Black aspergilli, Aspergillus tubingensis, Genomics, Secondary metabolism

\section{Background}

Filamentous fungi of the Aspergillus genus, and especially black Aspergilli, have a worldwide distribution and occur on a large variety of substrates. Due to their versatile metabolism, those fungi are one of the most prolific sources of enzymes, organic acids and secondary metabolites (SM) with biomedical and biotechnological interests [1-4]. Already described as non-mycotoxins producer in comparison to A. niger [5-9], A. tubingensis, which is part of the $A$. niger clade of the black Aspergilli, represents a good alternative for metabolites production in industrial fermentation and is already used for some applications [10-12].

\footnotetext{
* Correspondence: florence.mathieu@ensat.fr

${ }^{1}$ Université de Toulouse, Laboratoire de Génie Chimique, UMR 5503 CNRS/ INPT/UPS, INP-ENSAT, 1, avenue de I'Agrobiopôle, 31326 Castanet-Tolosan, France

Full list of author information is available at the end of the article
}

A. niger clade is exploited since 1923 for industrial fermentation, production of enzymes / heterologous proteins ( $\alpha$-amylases, cellulase, pectinase) and organic acids (citric acid) used in food, cosmetic and pharmaceutical preparations $[1,13,14]$. Besides, strains of this clade are remarkable producers of secondary metabolites of industrial, agricultural and economic importance. For example, they have the potential to produce compounds such as asperazine, which has antibiotic properties, or Naphtho-Gamma-Pyrones (NGPs), showing antioxidant, anticancer or antibiotic properties [1, 2]. However, they can also produce mycotoxins such as ochratoxin A (OTA) and fumonisin $B_{2}$, which are major concerns in risk assessment in the food chain [5].

It was recently suggested that availability of Aspergillus genomic sequences greatly facilitates secondary metabolites (SM) biosynthesis characterization, as expression of 
most of them is cryptic [15]. Genomic studies allow identification of numerous genes putatively involved in secondary metabolites production. Indeed, filamentous fungi possess a great number of biosynthetic genes such as polyketide synthase (PKS) or non-ribosomal peptide synthase (NRPS). These biosynthetic genes are often clustered with various enzymes-coding-genes (hydroxylase, methyltransferase, cytochrome P450) [1, 16, 17]. However, SM clusters genomic diversity does not reflect the metabolite profiles obtained under laboratory culture conditions, suggesting that a majority of SM biosynthetic pathways are transcriptionally silenced. Those silent biosynthetic pathways could be a rich source of chemically diverse compounds with outstanding potential for industry [16]. Today genomic studies are the best way to get a global view of fungal SM clusters and also offer a good view for their process optimization in industrial production and application.

Sequencing the genomes of a large number of strains from the same species, the same clade or the same genus, enables to run comparative studies on both genomic and biochemical levels [17]. These comparative studies will allow the characterization of biosynthetic pathways based on genes present / absent in organisms regarding their potentiality to produce one specific type of SM. For example, this methodology was applied for OTA biosynthesis genes cluster determination in $A$. carbonarius by sequencing and comparing the genomes of a producing and a non-producing strains $[18,19]$.

In this study, we report the draft genome of A. tubingensis G131 isolated from a French Mediterranean vineyard. This genome has been compared to the recently available A. tubingensis CBS 134.48 [20], A. niger CBS 513.88 [13] and A. kawachii IFO 4308 [21]. Those strains were chosen for their industrial applications. Indeed, $A$. niger CBS 513.88 is an industrial enzyme producer and A. kawachii IFO 4308 is a citric acid industrial producer used in koji fermentation for shochu beverage preparation [13, 21]. Those strains cover all types of metabolites that are industrially produced by black Aspergilli. This study focuses on the SM production potential of $A$. tubingensis G131, after a general genome comparison. Results presented here show that the strain does not contain SM clusters required for OTA and fumonisins production, as identified in $A$. niger CBS 513.88 [13, 22]. A complete review of the putative SM gene biosynthetic clusters is also reported. A biochemical analysis shows that A. tubingensis G131 produces asperazine and NGPs under laboratory conditions. The comparative genomic analyses conducted on the $A$. niger clade allowed us proposing putative SM clusters involved in asperazine and NGPs production $[1,23,24]$.

\section{Results and discussion}

\section{Genome sequencing}

The genome of A. tubingensis G131 isolated from a French Mediterranean vineyard [25], with black aspergilli morphological characteristics, was sequenced using Illumina MiSeq technology with a coverage of 143.6X. The genome assembly is approximately $35,18 \mathrm{Mb}$ long and includes 192 scaffolds with an average length of 183,235 bp (Table 1).

According to BUSCO analysis [26], the assembly of $A$. tubingensis G131 genome is robust. Indeed $98,8 \%$ of the 4046 groups of genes required for the correct assembly of eurotiomycetes were present in A. tubingensis scaffolds assembly (BUSCO results are available in Additional file 1: Table S1).

The genome size of $A$. tubingensis $\mathrm{G} 131(35,18 \mathrm{Mb})$ is equivalent to the genome size of $A$. tubingensis CBS 134.48 (35,15 Mb, Table 1) but larger than the A. niger CBS $513.88(34.02 \mathrm{Mb})$ genome and smaller than the $A$. kawachii IFO 4308 (36,6 Mb) genome [13, 20, 27]. The average shared identity at a nucleic acid level was obtained with ANI calculator [28]. It suggests that the sequenced strain is genetically closer to $A$. tubingensis $\mathrm{CBS}$ 134.48 (98.6\%) then to A. kawachii IFO 4308 (93,75\%) and finally to $A$. niger CBS $513.88(87.62 \%)$ but is really far from A. carbonarius ITEM 5010 (80.36\%) which is

Table 1 Genome characteristics and predicted features of the assembled A. tubingensis strains

\begin{tabular}{lll}
\hline Genome & A. tubingensis & A. tubingensis \\
& G131 (this study) & 334.48 (de Vries et al. 2017) \\
\hline Number of scaffolds & 192 & $4,803,603 \mathrm{bp}$ \\
Length of the largest scaffolds & $2,380,764 \mathrm{bp}$ & $1,065,035 \mathrm{bp}$ \\
Average length of scaffolds & $183,235 \mathrm{bp}$ & $35,15 \mathrm{Mb}$ \\
Total length of scaffolds & $35,18 \mathrm{Mb}$ & $125.7 \times$ \\
Sequence Read Coverage & $143.6 \mathrm{X}$ & $49.18 \%$ \\
$\mathrm{G}+\mathrm{C}$ content (\%) & $50.22 \%$ & 12,322 \\
Number of predicted coding genes & 10,994 & $475 \mathrm{aa}$ \\
\hline
\end{tabular}


also part of the $A$. niger clade. As the genome was closer to $A$. tubingensis CBS 134.48 on a nucleic acid level, a dot plot analysis against this strain was made to order scaffolds assembly (dot plot graphic is available in Additional file 1: Fig. S1). The dot plot alignment does not show any major evolutive event between the two $A$. tubingensis strains.

\section{Taxonomy}

A. tubingensis G131 was isolated on a survey on the occurrence of NGPs and OTA producing fungi in grapes [25]. Based on morphological characteristics, this strain was first classified as an A. niger strain. Actually, A. niger and $A$. tubingensis are the two most common species found in the black Aspergilli. However, due to their close morphological characteristics and often insufficient molecular identification, A. tubingensis strain is, most of the time, misidentified as $A$. niger [1].

Colony diameter of A. tubingensis G131 is superior at $85 \mathrm{~mm}$ after 7 days cultures at $28{ }^{\circ} \mathrm{C}$ (Fig. 1a). On these conditions, the filamentous fungus displays high sporulation. Conidial heads are dark brown to black, commonly abundant and slightly floccose as already described in A. tubingensis sp. [29]. Conidiophores $(70-90 \mu \mathrm{m})$ seem to have a limited surface granulation (Fig. 1b). Conidia have a size range of 3-5 $\mu \mathrm{m}$ with a spiny appearance as already described by
Samson et al. [29]. However, these morphological characteristics are really close to those of A. niger, explaining the confusion previously made by Bouras et al. [25].

The phylogenetic analysis performed in this study used several reference genes (rpb2, benA, cam1) and revealed the close relationship between the sequenced strain and other A. tubingensis strains [29]. The multilocus analysis was performed on our isolate with 37 reference strains from black Aspergilli (NCBI accession number available in Additional file 1: Table S2). These reference genes were chosen for their percentage of variable and parsimony informative sites per locus as described by Jurjevic et al. [30]. Phylogenetic analysis was conducted first on the three single locus alignments, then the three partial gene sequences were combined in a unique alignment (Fig. 2). The tree with the highest log likelihood is shown. The phylogenic trees of each single locus (Additional file 1: Figures S2, S3, S4) or of the combined loci show almost the same topology. Besides, these results fulfill the requirements of genealogical concordance and phylogenetic species recognition [31], identifying the sequenced strain as an A. tubingensis. Besides, phylogenetic results confirm than the sequenced strain is genetically closer to $A$. kawachii IFO 4308 than to $A$. niger CBS 513.88.

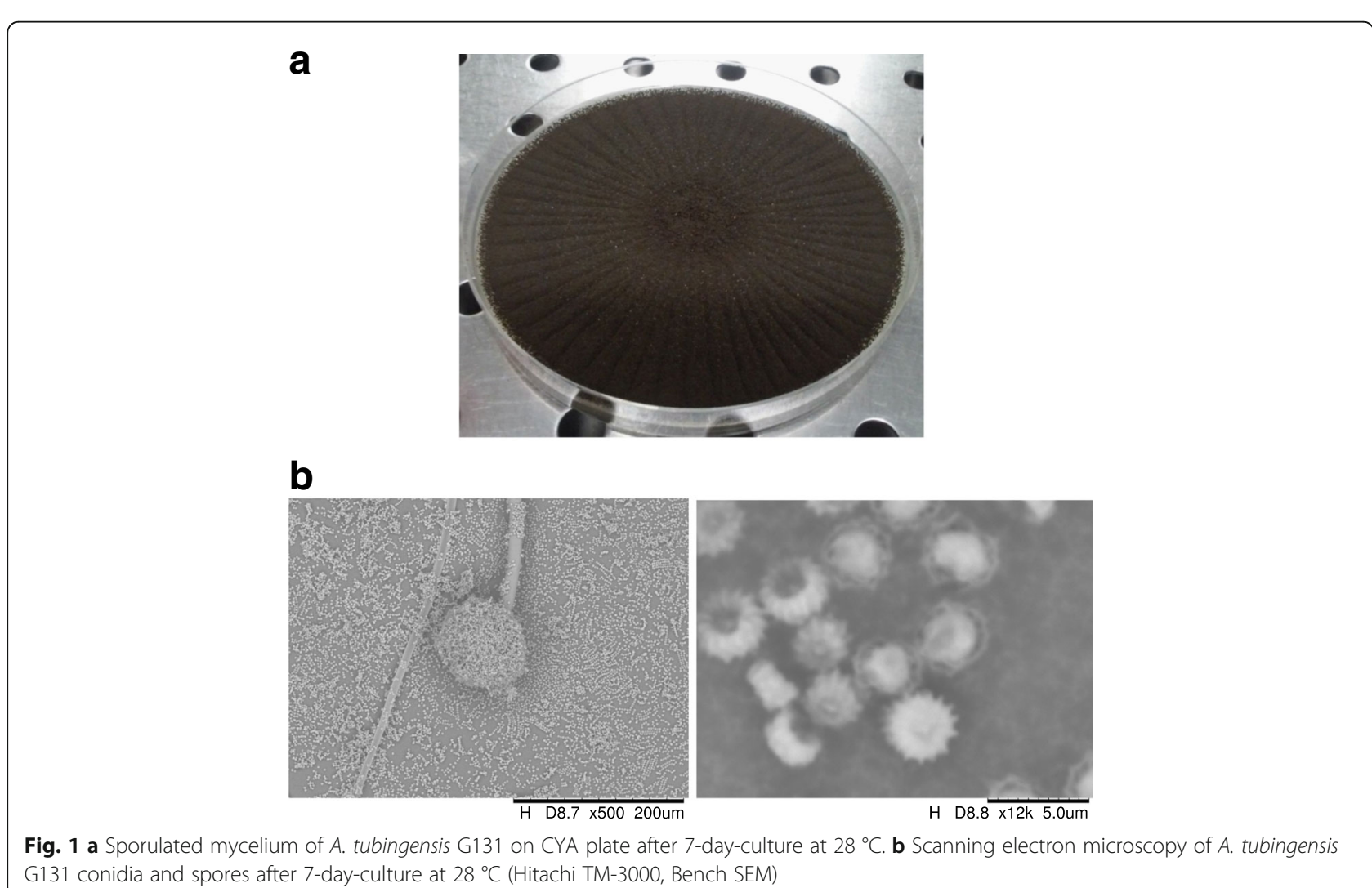




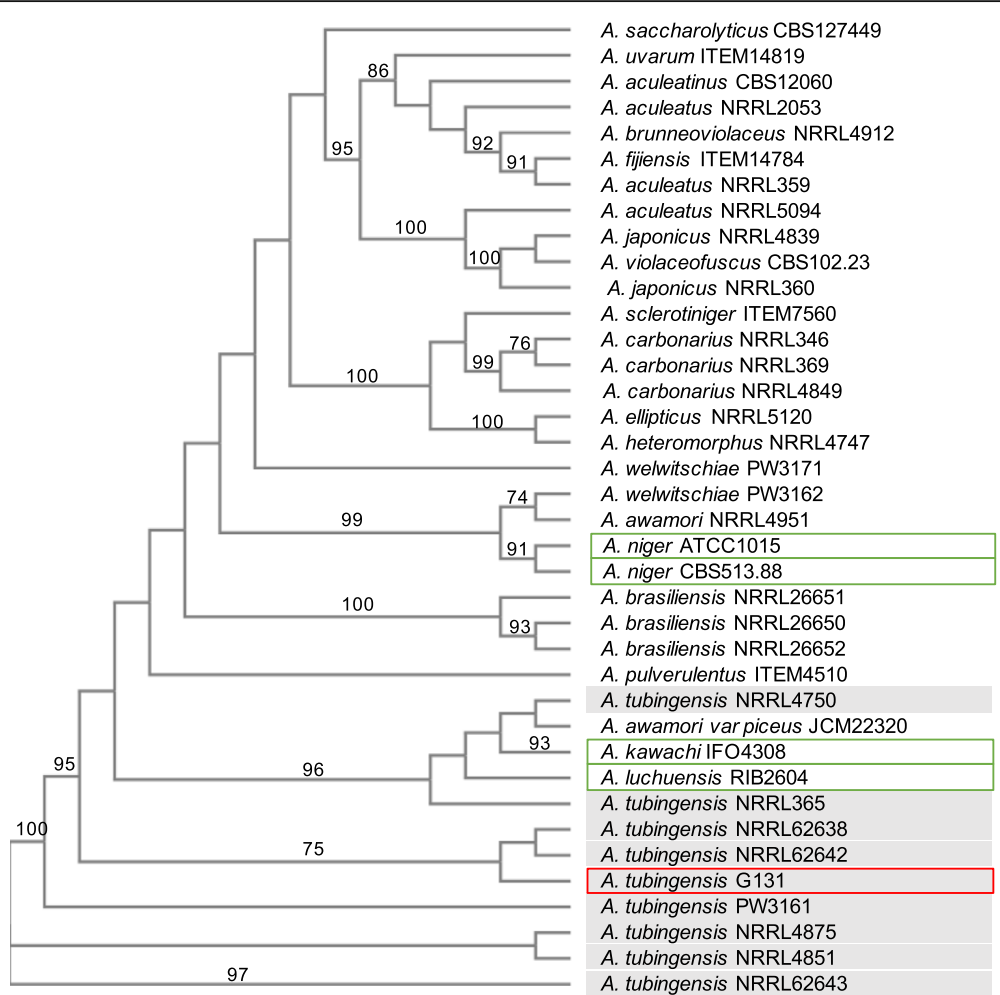

Fig. 2 Phylogenetic tree produced from the combined sequence data of three loci (rpb2, benA, cam 1) of 38 strains of uniseriate black Aspergilli. Numbers above branches are bootstrap values. Only those above $70 \%$ are indicated. The evolutionary history was inferred using the NeighbourJoining method computed with the Maximum Likelihood Evolutionary method. Grey background highlighted A. tubingensis strains; Green frame highlighted the genome used for the comparative analysis of this study; Red frame highlighted the genome sequenced in this study

\section{Genome annotation}

Genome annotation was performed with Augustus gene prediction software [32]. The annotation predicts 10,994 coding genes, which is less than what is described for the other fungi from the $A$. niger clade: $A$. tubingensis CBS 134.48 (12322), A. niger CBS 513.88 (14097) and $A$. kawachii IFO 4308 (11475) [13, 20, 21]. The low number of predicted coding genes for $A$. tubingensis G131 could be linked to the prediction methodology. Indeed, RNA sequencing was also used for genes prediction in the three other genomes. We observe a difference of 1328 predicted genes between A. tubingensis CBS 134.48 and A. tubingensis $\mathrm{G} 131$. Such a discrepancy was already observed between $A$. niger CBS 513.88 and A. niger ATCC 1015, which showed a difference of 2882 predictive proteins [14]. Authors affirmed that the difference between the two $A$. niger strains is due to "overprediction in CBS 513.88 / underprediction in ATCC 1015". To confirm such a hypothesis in the case of $A$. tubingensis strains (A. tubingensis CBS 134.48 and A. tubingensis G131), we runned the Augustus software (same parameters than for A. tubingensis G131) for proteins prediction in $A$. tubingensis CBS 134.48 genome assembly and found 10,652 predicted genes in comparison to 10,994 predicted genes in A. tubingensis G131. So, this small difference clearly argued for the overprediction/underprediction hypothesis.

Predicted proteins were annotated through homology search with NCBI BLAST ( $\mathrm{nr} / \mathrm{nt}$ database), Interproscan and Gene Ontology using Blast2GO software [33] (Blast2Go results summary are available in Additional file 1: Figure S5). The average length of predicted proteins is 524 amino acids (Table 1). This value is higher than the average protein length of other black Aspergilli: A. tubingensis CBS 134.48 (475aa), A. niger CBS 513.88 (442,5 aa) $A$. kawachii IFO $4308(500,1$ aa) [13, 20, 21]. In any case, those results are in agreement with Tiessen et al. who have shown that average protein length in fungi is 487 aa [34].

Eukaryotic orthologous groups [35] (KOG) functional classification of the four compared genomes is shown in Fig. 3a (raw data are available in Additional file 1: Table S3). Four main categories can be distinguished: intracellular processes, metabolism, information storage / processing and function poorly characterized. Results show that function repartition is approximately the same in the four compared genomes. KOG analyses reveal that most of the genes are involved in metabolism: amino acid $(\approx 437$ proteins / strain), carbohydrates $(\approx 771$ proteins / strain), lipid ( $\approx 482$ proteins / strain) or secondary metabolites $(\approx 422$ proteins / strain). In the case of 


\section{a}

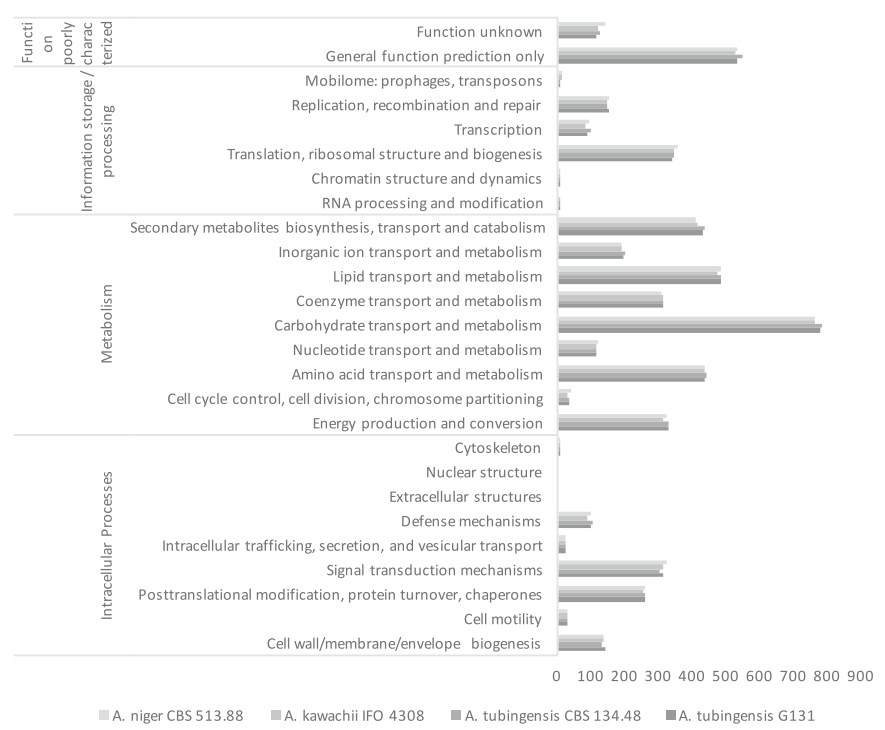

b

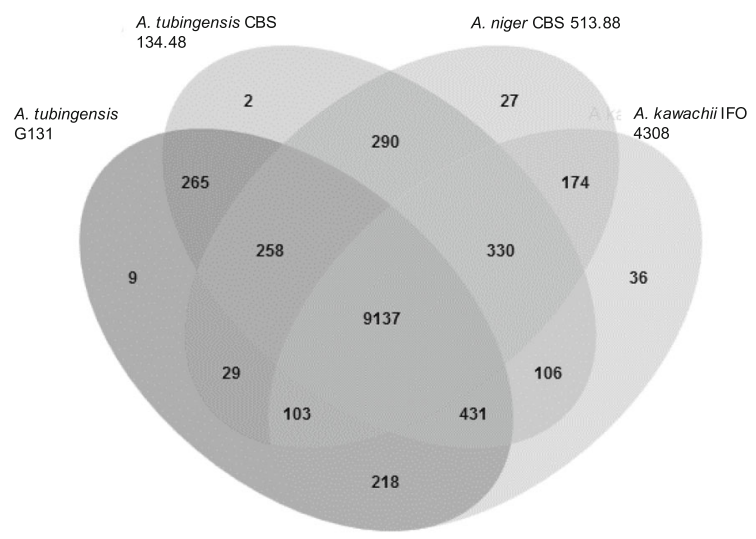

Fig. 3 Global comparative genomic analyses of A. tubingensis G131, A. tubingensis CBS 134.48, A. niger CBS 513.88 and A. kawachii IFO 4308 a. Histogram of KOG distribution of predicted proteins from each genome. $\mathbf{b}$. Venn diagram distribution of orthologous gene clusters from the four genomes obtain with OrthoMCL analysis

A. niger clade, genes involved in metabolism represent $52 \%$ of the KOG classification which might support the advantages of this type of strains for industrial application.

OrthoMCL analysis compares amino acid sequences of different genomes through BlastP analyses, clustering them according to their identity (threshold: 50\% similarity) [36]. An OrthoMCL analysis was performed on A. tubingensis G131, A. tubingensis CBS 134.48, A. niger CBS 513.88 and $A$. kawachii IFO 4308. Venn diagram of the OrthoMCL results (Fig. 3b) shows a strong homology between the four strains of the A. niger clade as 9137 orthologous clusters contain at least one amino acid sequence of each strain. The specific genes, showing no orthology or paralogy relationships between A. tubingensis G131, A. tubingensis CBS 134.48, A. niger CBS 513.88 and A. kawachii IFO 4308 were 344, 1331, 1355 and 649 respectively. Moreover, 276 clusters were specific to $A$. tubingensis strains and among them 2 were specific to $A$. tubingensis CBS 134.48 (proteins of unknown function) and 9 were specific to $A$. tubingensis G131. Among them, 6 were of unknown functions, 1 was a putative carboxylesterase, 1 was a DEAD-box RNA helicase and 1 was a putative transposase. Among the 265 orthologous clusters specific to both $A$. tubingensis, 46 show useful functions for SM synthesis: cytochromes P450 (10), dehydrogenases (6), mono/dioxygenases (6), hydrolases (5), aldehyde reductases (5), oxidases (4), carboxylesterases (3), AcetylCoA synthases (2), decarboxylase (2), methyltransferases (2), methylesterase (1). Among the 9 orthologous clusters present in A. tubingensis G131, no homology with other fungal species genes was found. Moreover, 334 predictive proteins from $A$. tubingensis G131 were not sorted in orthologous clusters and were specific of the studied strain. 23\% (78) of these specific proteins seems to have an exogenous origin from different fungal species such as Penicillium sp., Metarhizium sp., Aspergillus sp., Trichoderma sp. Interestingly, among those 78 exogenous acquisitions, 23 predictive proteins seem to have been acquired from Penicillium chrysogenum and 10 from Aspergillus oryzae. As those two genomes (Penicillium chrysogenum, Aspergillus oryzae) were only assembled in contigs, it is impossible to conclude if they are the result of a single or multiple chromosomal insertion over time.

Considering that many industrial purposes require the production of extracellular enzymes, a secretome analysis was made to estimate the percentage of secreted proteins in each studied genome. Results show that approximately, $10 \%$ of each predicted proteome encode secreted proteins for A. tubingensis G131 (1107), A. tubingensis CBS 134.48 (1391), A. kawachii IFO 4308 (1204) and $A$. niger CBS 513.88 (1307). Results show a core secreted proteome composed of 774 protein orthologous clusters between the four strains (at least 1 putative secreted protein from each Aspergillus sp. is present in a cluster - Venn diagram is available in Additional file 1: Figure S6). Regarding biological process, 25,3\% of the core secretome acts in secondary metabolic processes, $3,8 \%$ acts in cell wall formation and $3,8 \%$ acts as response to external stimulus. Besides, $44 \%$ of this core 
secretome shows a SM synthesis function such as hydrolase $(28,7 \%)$, peptidase $(5,9 \%)$, oxidoreductase $(5,2 \%)$ or transferase $(4,1 \%)$. Finally, $32,5 \%$ of the core secretome is required in fungal cell wall or membrane formation and $15,5 \%$ is extracellular. Among the extracellular proteins, proteins with SM synthesis functions are mostly found. Regarding secretome specifity of A. tubingensis G131, we observe 59 unique secreted proteins. $25 \%$ of those proteins seem to have been acquired from various fungal source such as Penicillum spp., Colletotrichum spp., Fusarium spp. or Neosartorya spp.. Among those 59 unique secreted proteins, only 3 functions have been found: polysaccharide deacetylase, carboxylesterase and short-chain dehydrogenase reductase. Other putative functions remain unknown as no conserved domain is observable.

Focus on Aspergillus tubingensis G131 secondary metabolism General observation on secondary metabolites biosynthesis Regarding dot-plot analysis of A. tubingensis G131 against $A$. tubingensis CBS 134.48 (Additional file 1: Figure S1), A. kawachii IFO 4308 or A. niger CBS 513.88 genome assembly, the only genomic re-arrangement observed is a $0.7 \mathrm{Mb}$ inversion observed in $A$. niger CBS 513.88. No duplication, insertion or deletion event were observable in the analysis. It seems that genomic variability between strains and thus their potential to produce specific metabolites were only caused by genomic mutations or genes acquisition / deletion but not chromosomal re-arrangement. This result is in agreement with Lind et al. (2017) which argued that the identity and total number of SM clusters can vary between very closely related species, as for the four studied Aspergillus sp., whose genomes exhibit very high sequence and synteny conservation [37].

SM clusters prediction in A. tubingensis G131 was done using two available software packages: AntiSMASH and SMURF [38, 39]. AntiSMASH, based on conserved domains search on genome assembly, predicts 81 putative clusters. While SMURF based on fungal clusters comparison from predicted proteins, predicts 66 putative clusters. Inglis et al. [15] already described that both softwares have efficient algorithm for SM clusters prediction. However, there are disparities between predicted cluster boundaries. Results need to be manually refined by taking the farthest one. In A. tubingensis G131, 80 SM clusters are predicted after manual refinement. The list of $A$. tubingensis G131 putative SM clusters and their genomic coordinates are available in Additional file 1: Table S4.

The predicted SM clusters of $A$. tubingensis G131 are defined according to their "backbone enzymes" that generate the carbon skeleton of the putative SM. In $A$. tubingensis G131, most of the "backbone enzymes" are polyketide synthase (PKS) or non-ribosomal peptides synthase (NRPS). 31 SM clusters contain sequence coding for a PKS / PKS-like enzymes, 29 SM clusters contain sequence coding for a NRPS / NRPS-like enzymes and 13 SM clusters are hybrid clusters containing sequence coding for both PKS and NRPS enzymes (Table 2). The remaining SM clusters seem to be required in terpene/terpenoid metabolites production as the "backbone enzyme" is a terpene cyclase (9 SM clusters) or a dimethylallyl tryptophan synthase (2 SM clusters). Moreover, we observe that A. tubingensis G131 possesses a greater number of PKS / NRPS hybrid SM clusters (13) than the other strains (Table 2).

Genomic sequences of $A$. tubingensis G131 SM clusters were individually blasted against $A$. tubingensis CBS 134.48, A. niger CBS 513.88 and A. kawachii IFO 4308 using NCBI blast on whole genome shotgun (WGS) NCBI data (Table 3). In this study, a SM cluster was considered as highly conserved in another $A$. niger clade strain when BLAST coverage $>80 \%$ and identity $>80 \%$. This characterization allows us to discriminate four types of SM clusters: (i) highly conserved in A. niger, A. tubingensis, A. kawachii (36 SM clusters); (ii) highly conserved in

Table 2 Distribution of types of predicted SM clusters, according to their backbone enzymes, predict in A. tubingensis G131, A. tubingensis CBS 134.48, A. niger CBS 513.88 and A. kawachii IFO 4308

\begin{tabular}{lllll}
\hline & A. tubingensis & A. tubingensis & A. niger \\
G131 (this study) & $\begin{array}{l}\text { CBS 134.48 (de } \\
\text { Vries et al., 2017) }\end{array}$ & $\begin{array}{l}\text { A. kawachii } \\
\text { (Andersen et al., 2011) }\end{array}$ & $\begin{array}{l}\text { IFO 4308 } \\
\text { (Yamada et al., 2016) }\end{array}$ \\
\hline DMAT & 2 & 2 & 2 & 2 \\
Hybrid & 13 & 7 & 6 & 6 \\
NRPS & 14 & 14 & 14 & 15 \\
NRPS-like & 11 & 19 & 15 & 17 \\
PKS & 28 & 31 & 29 & 33 \\
PKS-like & 3 & 6 & 5 & 5 \\
TC & 9 & 6 & 6 & 6 \\
Total & 80 & 85 & 77 & 84 \\
\hline
\end{tabular}

DMAT dimethylallyl tryptophan synthase, Hybrid PKS / NRPS, TC Terpene cyclase 
Table 3 Alignment of SM clusters predicted in A. tubingensis G131 with genome assembly of A. tubingensis CBS 134.48, A. niger CBS 513.88 and A. kawachii IFO 4308. cov: coverage; Id: Identity

\begin{tabular}{|c|c|c|c|c|c|c|c|c|c|c|}
\hline \multirow[t]{3}{*}{ Group } & \multirow[t]{3}{*}{ Cluster } & \multirow[t]{3}{*}{ Type } & \multirow[t]{3}{*}{ Size (Mb) } & \multirow{3}{*}{$\begin{array}{l}\text { Similarity to } \\
\text { known clusters }\end{array}$} & & & & & & \\
\hline & & & & & \multicolumn{2}{|c|}{$\begin{array}{l}\text { A. tubingensis } \\
\text { CBS } 134.48\end{array}$} & \multicolumn{2}{|c|}{$\begin{array}{l}\text { A. niger } \\
\text { CBS } 513.88\end{array}$} & \multicolumn{2}{|c|}{$\begin{array}{l}\text { A. kawachii } \\
\text { IFO } 4308\end{array}$} \\
\hline & & & & & $\begin{array}{l}\text { Cov } \\
(\%)\end{array}$ & Id (\%) & $\overline{\operatorname{cov}(\%)}$ & $\overline{l d}(\%)$ & $\operatorname{cov}(\%)$ & Id (\%) \\
\hline \multirow{36}{*}{$\begin{array}{l}\text { Highly conserved } \\
\text { A. tubingensis A. niger } \\
\text { A. kawachii }\end{array}$} & Cluster 3 & NRPS & 45,6 & & 99 & 98 & 97 & 87 & 99 & 94 \\
\hline & Cluster 6 & Terpene & 21,3 & & 90 & 96 & 93 & 86 & 100 & 92 \\
\hline & Cluster 7 & PKS & 56,2 & & 97 & 91 & 93 & 89 & 97 & 95 \\
\hline & Cluster 9 & PKS - Like & 12,6 & & 100 & 99 & 98 & 90 & 100 & 96 \\
\hline & Cluster 13 & PKS & 37,7 & & 99 & 98 & 84 & 85 & 99 & 92 \\
\hline & Cluster 16 & PKS & 42,0 & & 100 & 100 & 80 & 85 & 97 & 92 \\
\hline & Cluster 17 & PKS & 51,9 & & 100 & 99 & 91 & 86 & 99 & 94 \\
\hline & Cluster 18 & NRPS & 61,9 & & 98 & 99 & 81 & 91 & 88 & 95 \\
\hline & Cluster 23 & NRPS & 62,7 & Aflatrem (cov: 11\%) & 97 & 96 & 82 & 88 & 95 & 93 \\
\hline & Cluster 24 & NRPS & 41,5 & & 99 & 97 & 81 & 87 & 93 & 92 \\
\hline & Cluster 25 & NRPS / PKS & 77,7 & & 98 & 98 & 84 & 89 & 96 & 95 \\
\hline & Cluster 29 & NRPS-Like & 50,4 & & 99 & 99 & 85 & 92 & 93 & 96 \\
\hline & Cluster 32 & PKS & 35,6 & & 99 & 99 & 92 & 88 & 97 & 96 \\
\hline & Cluster 33 & PKS & 48,0 & & 95 & 98 & 80 & 90 & 92 & 94 \\
\hline & Cluster 34 & Terpene & 22,0 & & 99 & 96 & 98 & 90 & 99 & 95 \\
\hline & Cluster 35 & NRPS-Like & 63,0 & & 99 & 99 & 88 & 91 & 98 & 96 \\
\hline & Cluster 36 & PKS & 50,5 & & 100 & 99 & 97 & 90 & 99 & 95 \\
\hline & Cluster 37 & Terpene & 22,1 & & 99 & 98 & 88 & 89 & 99 & 94 \\
\hline & Cluster 38 & NRPS-Like & 73,4 & & 94 & 98 & 92 & 92 & 93 & 94 \\
\hline & Cluster 39 & NRPS-Like & 54,8 & & 100 & 99 & 96 & 90 & 99 & 96 \\
\hline & Cluster 42 & NRPS & 47,2 & & 100 & 98 & 95 & 89 & 98 & 95 \\
\hline & Cluster 43 & NRPS & 44,0 & & 98 & 97 & 85 & 87 & 97 & 95 \\
\hline & Cluster 45 & PKS & 59,3 & & 99 & 96 & 81 & 91 & 93 & 93 \\
\hline & Cluster 49 & PKS & 68,4 & & 97 & 95 & 80 & 88 & 95 & 96 \\
\hline & Cluster 50 & PKS & 46,5 & & 100 & 98 & 92 & 90 & 100 & 95 \\
\hline & Cluster 52 & PKS & 83,8 & & 99 & 96 & 84 & 85 & 98 & 94 \\
\hline & Cluster 53 & Terpene & 21,6 & & 100 & 98 & 96 & 87 & 99 & 95 \\
\hline & Cluster 54 & PKS & 44,7 & & 99 & 99 & 97 & 90 & 99 & 94 \\
\hline & Cluster 56 & PKS / NRPS & 41,3 & & 98 & 97 & 86 & 85 & 91 & 92 \\
\hline & Cluster 62 & Terpene & 20,8 & & 100 & 99 & 88 & 90 & 99 & 96 \\
\hline & Cluster 63 & NRPS-Like & 56,8 & & 99 & 99 & 93 & 90 & 98 & 95 \\
\hline & Cluster 58 & NRPS & 77,8 & & 100 & 99 & 85 & 83 & 89 & 91 \\
\hline & Cluster 67 & NRPS-Like & 40,9 & & 100 & 99 & 91 & 90 & 97 & 95 \\
\hline & Cluster 69 & Indole / DMAT & 21,4 & & 100 & 99 & 84 & 86 & 99 & 93 \\
\hline & Cluster 74 & NRPS & 75,9 & & 100 & 99 & 97 & 89 & 99 & 95 \\
\hline & Cluster 77 & NRPS & 54,6 & & 99 & 99 & 97 & 91 & 99 & 98 \\
\hline \multirow{4}{*}{$\begin{array}{l}\text { Highly conserved } \\
\text { A. tubingensis } \\
\text { A. kawachii }\end{array}$} & Cluster 1 & PKS & 46,8 & & 100 & 99 & 50 & 91 & 97 & 95 \\
\hline & Cluster 2 & Indole / DMAT & 21,5 & Notoamide (cov: 20\%) & 97 & 97 & 77 & 89 & 93 & 94 \\
\hline & Cluster 4 & PKS & 41,8 & Fumonisin (cov: 31\%) & 100 & 99 & 54 & 81 & 92 & 90 \\
\hline & Cluster 5 & PKS & 42,4 & & 86 & 98 & 74 & 88 & 92 & 96 \\
\hline
\end{tabular}


Table 3 Alignment of SM clusters predicted in A. tubingensis G131 with genome assembly of A. tubingensis CBS 134.48, A. niger CBS 513.88 and A. kawachii IFO 4308. cov: coverage; Id: Identity (Continued)

\begin{tabular}{|c|c|c|c|c|c|c|c|c|c|c|}
\hline \multirow[t]{3}{*}{ Group } & \multirow[t]{3}{*}{ Cluster } & \multirow[t]{3}{*}{ Type } & \multirow[t]{3}{*}{ Size (Mb) } & \multirow{3}{*}{$\begin{array}{l}\text { Similarity to } \\
\text { known clusters }\end{array}$} & \multirow{2}{*}{\multicolumn{2}{|c|}{$\begin{array}{l}\text { A. tubingensis } \\
\text { CBS } 134.48\end{array}$}} & \multirow{2}{*}{\multicolumn{2}{|c|}{$\begin{array}{l}\text { A. niger } \\
\text { CBS } 513.88\end{array}$}} & \multirow{2}{*}{\multicolumn{2}{|c|}{$\begin{array}{l}\text { A. kawachii } \\
\text { IFO } 4308\end{array}$}} \\
\hline & & & & & & & & & & \\
\hline & & & & & $\begin{array}{l}\text { Cov } \\
(\%)\end{array}$ & Id (\%) & $\operatorname{cov}(\%)$ & Id (\%) & $\operatorname{cov}(\%)$ & Id (\%) \\
\hline & Cluster 10 & Terpene & 22,9 & & 88 & 98 & 72 & 88 & 98 & 94 \\
\hline & Cluster 11 & NRPS-Like & 42,7 & & 100 & 99 & 74 & 93 & 94 & 92 \\
\hline & Cluster 12 & PKS & 29,8 & & 100 & 99 & 26 & 82 & 66 & 95 \\
\hline & Cluster 15 & NRPS-Like & 54,5 & & 100 & 99 & 79 & 85 & 89 & 93 \\
\hline & Cluster 19 & NRPS / PKS & 71,4 & & 98 & 99 & 43 & 89 & 86 & 97 \\
\hline & Cluster 20 & PKS & 46,7 & & 98 & 98 & 60 & 86 & 95 & 94 \\
\hline & Cluster 22 & PKS / NRPS & 66,5 & & 100 & 99 & 66 & 88 & 85 & 91 \\
\hline & Cluster 26 & PKS & 40,9 & & 100 & 99 & 67 & 83 & 81 & 92 \\
\hline & Cluster 31 & PKS & 60,1 & & 97 & 97 & 75 & 83 & 91 & 92 \\
\hline & Cluster 40 & Other & 29,9 & & 86 & 99 & 65 & 89 & 80 & 93 \\
\hline & Cluster 41 & PKS & 45,3 & & 96 & 99 & 68 & 91 & 91 & 93 \\
\hline & Cluster 47 & PKS & 57,7 & & 100 & 99 & 51 & 83 & 83 & 92 \\
\hline & Cluster 51 & NRPS / PKS & 102,4 & Stigmatellin (cov: 30\%) & 96 & 97 & 76 & 84 & 82 & 93 \\
\hline & Cluster 55 & NRPS-Like & 53,8 & & 85 & 96 & 69 & 89 & 87 & 95 \\
\hline & Cluster 57 & PKS & 42,1 & & 100 & 99 & 53 & 85 & 95 & 92 \\
\hline & Cluster 60 & PKS / NRPS & 35,1 & & 90 & 96 & 77 & 87 & 86 & 92 \\
\hline & Cluster 64 & PKS & 70,4 & & 99 & 97 & 78 & 83 & 95 & 93 \\
\hline & Cluster 66 & PKS / NRPS & 63,0 & & 100 & 99 & 71 & 88 & 95 & 92 \\
\hline & Cluster 68 & NRPS-Like & 33,8 & & 100 & 99 & n.d. & n.d. & 83 & 91 \\
\hline & Cluster 70 & NRPS-Like & 52,8 & & 100 & 99 & 51 & 83 & 84 & 91 \\
\hline & Cluster 72 & NRPS / PKS & 43,8 & & 100 & 99 & 15 & 81 & 85 & 91 \\
\hline & Cluster 73 & NRPS & 45,7 & & 99 & 99 & 59 & 88 & 82 & 91 \\
\hline & Cluster 75 & Terpene / PKS & 50,7 & & 100 & 99 & 76 & 92 & 94 & 94 \\
\hline & Cluster 76 & NRPS & 45,5 & & 99 & 97 & 75 & 87 & 97 & 92 \\
\hline \multirow{9}{*}{$\begin{array}{l}\text { Highly conserved } \\
\text { A. tubingensis }\end{array}$} & Cluster 8 & NRPS / PKS & 54,0 & Isoflavipucine (cov: 12\%) & 100 & 99 & 61 & 95 & 25 & 97 \\
\hline & Cluster 14 & NRPS / PKS & 66,2 & & 100 & 99 & 42 & 86 & 60 & 94 \\
\hline & Cluster 27 & NRPS & 73,4 & & 100 & 99 & 51 & 90 & 60 & 95 \\
\hline & Cluster 30 & NRPS & 59,6 & & 87 & 99 & 58 & 86 & 75 & 93 \\
\hline & Cluster 46 & PKS / NRPS & 60,7 & Shanorellin (cov: 28\%) & 100 & 99 & 53 & 85 & 68 & 92 \\
\hline & Cluster 48 & PKS & 47,9 & & 97 & 97 & 58 & 82 & 78 & 91 \\
\hline & Cluster 59 & PKS & 46,6 & & 100 & 99 & 57 & 82 & 70 & 90 \\
\hline & Cluster 61 & NRPS / PKS & 79,1 & & 100 & 99 & 75 & 87 & 69 & 93 \\
\hline & Cluster 65 & NRPS & 56,7 & & 83 & 98 & 41 & 84 & 70 & 93 \\
\hline \multirow{7}{*}{$\begin{array}{l}\text { Specific to } A . \\
\text { tubingensis G131 }\end{array}$} & Cluster 21 & PKS & 82,5 & Aflavarin (cov: 40\%) & 68 & 95 & 57 & 86 & 77 & 92 \\
\hline & Cluster 28 & PKS-Like & 15,3 & & 54 & 89 & 48 & 82 & 54 & 92 \\
\hline & Cluster 44 & PKS & 32,8 & TAN-1612 (cov: 80\%) & 71 & 96 & 75 & 89 & 72 & 92 \\
\hline & Cluster 71 & Terpene & 20,3 & & 76 & 98 & 65 & 86 & 76 & 93 \\
\hline & Cluster 78 & NRPS-Like & 27,5 & & 36 & 79 & 39 & 85 & 36 & 78 \\
\hline & Cluster 79 & Terpene & 22,1 & & n.d. & n.d. & 41 & 89 & 93 & 94 \\
\hline & Cluster 80 & PKS & 24,1 & & n.d. & n.d. & 30 & 93 & 64 & 88 \\
\hline
\end{tabular}


A. tubingensis and A. kawachii (28 SM clusters); (iii) highly conserved in A. tubingensis isolates (9 SM clusters); (iv) specific to $A$. tubingensis G131 (7 SM clusters).

Among the $7 \mathrm{SM}$ clusters specific to A. tubingensis G131, a particular attention should be focused on to cluster 44 (Table 3). According to AntiSMASH database, cluster 44 presents genes similarity of $80 \%$ with the biosynthetic cluster of an already known compound: TAN1612. This similarity suggests that $A$. tubingensis G131 could produce TAN-1612, a neuropeptide $\mathrm{Y}$ antagonist, already shown to be produced by A. niger CBS 513.88 and $A$. niger ATCC $1015[40,41]$. Cluster 44 shares genes similarity with all known anthracenone / naphthacenedione compounds produced by fungi: TAN-1612, viridicatumtoxin, asperthecin, neosartoricin and emericellin (Additional file 1: Figure S7). Those compounds show immunosuppressive properties [40]. Those results suggest that $A$. tubingensis G131 could produce anthracenone / naphthacenedione compounds with potential immunosuppressive properties.

The 6 other specific SM clusters present insertion of several $\mathrm{kb}$ with no homology in the NCBI nr/nt database. These insertions could confer new functionalities to the SM clusters or could lead to production of putative new secondary metabolites. The schematic representations of these SM clusters are available in Additional file 1: Figure S8. Cluster 21 is composed of 29 putative genes and contains at least 6 transporters and 2 transcription factors. The PKS enzyme is poorly conserved (BlastP maximum identity: 62\%) and is composed of 4 known PKS domains: KS - AT - PT (DH) - PP. The Product Template (PT) domain of the PKS as a dehydratase $(\mathrm{DH})$ function suggests Claisen cyclization of the natural product through loss of hydroxyl radicals. The presence of a gene coding a putative acyl-CoA dehydrogenase in the cluster suggests that one precursor of the putative natural compound could be acetyl-CoA. Moreover, the PP-binding domain of the PKS and a gene coding for a HMG-CoA reductase might suggest that a fatty acid chain could be attached and modified during the biosynthesis of the natural compound. Despite of this biosynthetic information, the structure of the putative compound cannot be estimated due to too many hypothetical proteins in the cluster.

\section{Focus on putative mycotoxins production}

The production of OTA and fumonisins by black Aspergilli has already been demonstrated, especially in A. niger $[1,22,25]$. OTA, for example, is produced in variable amounts depending on black Aspergilli species. A. carbonarius is known to consistently produce large amounts of OTA whereas only $6-10 \%$ of the $A$. niger produce it [1]. Black Aspergilli can also produce fumonisin B2 and fumonisin B4 [22, 42]. Based on the current knowledge about the biosynthesis of these mycotoxins, BLAST analyses were performed to check the mycotoxins production potentiality in A. tubingensis G131 strain, a methodology that was already described for different genomic works on Aspergillus sp. [27, 43, 44].

\section{Genomics features of putative OTA biosynthesis in $A$.} tubingensis G131 Through biochemical analyses, Bouras et al. [25] showed that A. tubingensis G131 does not produce OTA under certain conditions. According to literature, the main black Aspergilli OTA producer is A. carbonarius, which is also from the A. niger clade. Recently, the genome of $A$. carbonarius Acv3, an atoxigenic strain, was sequenced. Comparative genomic analyses with OTA producer $A$. carbonarius ITEM 5010 were performed focusing on genes known to be involved in OTA biosynthesis [18]. Cabañes et al. suggested that the atoxigenicity of $A$. carbonarius Acv3 could be linked to the high mutation rate observed in a specific PKS of this strain, affecting its function. It was shown that biosynthesis of OTA in A. niger CBS 513.88 is mediated by a PKS (An15g07920) which is clusterized with genes coding for a cytochrome P450 and an NRPS [13]. This cluster identified in $A$. niger CBS 513.88 genome is missing in A. niger ATCC 1015, a citric acid producer without OTA biosynthesis [14]. In addition, a 21-kb region of the A. niger CBS 513.88 OTA cluster is also absent in $A$. luchuensis NBRC 4314 and in A. kawachii IFO 4308 genomes, two strains which do not produce OTA $[21,27]$.

In this study, all the genetic information (genes and clusters sequences) available on OTA biosynthesis was blasted against $A$. tubingensis G131 genome (NCBI accession number and blast results are available in Additional file 1: Table S5). For example, A. tubingensis G131 does not contain an orthologue of An15g07920. However, although the backbone enzyme is missing, two genes from the putative OTA clusters in $A$. niger and $A$. welwitschiae have orthologues in A. tubingensis G131. These genes are coding for hypothetical proteins with none described key functions in OTA production. Moreover, based on their position in the genome, they are not included in one of the predictive SM cluster, suggesting that there is no backbone enzyme close to these genes. Genomic analyses suggest that A. tubingensis G131 could not produce OTA. This is in agreement with what was already published about the OTA non-productivity of $A$. tubingensis strains $[1,45]$.

In addition, one of the predicted proteins of $A$. tubingensis G131 shows strong identity (NCBI TBlastN Cov: 100\%; Id: 97.3\%) with an ochratoxinase from $A$. niger WK143 (NCBI accession: KJ854920.1), suggesting that the strain could be a putative biocontrol agent to limit OTA concentration in food chains. 
Genomics features of putative fumonisin biosynthesis in $A$. tubingensis G131 In contrast with OTA production, both $A$. niger CBS 513.88 and ATCC 1015 genomes include putative homologues of Fusarium verticillioides fumonisin genes and their production of Fumonisin B2 has been confirmed $[1,22,42]$. The fumonisin gene cluster of $A$. niger contains at least 14 fum genes [43]. A previous study showed that $A$. luchuensis NBRC 4314 genome only contains orthologues for fum 1 and fum 15 with high identity: 68 and 72\%, respectively [27]. Moreover, others FUM were predicted to be encoded by $A$. luchuensis, but the proteins only shared $20-43 \%$ identities and the orthologue genes of such proteins were distributed throughout the A. luchuensis genome. Susca et al. [43] suggested that fumonisin production was widespread among black Aspergilli. They demonstrated that nonrandom partial deletion of fumonisin cluster has occurred multiple times in several black Aspergilli genomes. Considering A tubingensis G131, the predictive SM cluster 4 shows partial homology with the $A$. niger fumonisins cluster. This cluster was identified by NCBI Blast $\mathrm{N}$ and it matches on approximately $13 \mathrm{~kb}$ among the $54 \mathrm{~kb}$ of the fumonisin cluster (Additional file 1: Figure S9). This represents only intergenic region (query coverage: $31 \%$; identity: $81 \%$, blast results are available in Additional file 1: Table S5). Except fum1 and fum15, none of the fum genes found in A. niger has an orthologue in A. tubingensis G131 (Additional file 1: Table S5). These results suggest that $A$. tubingensis G131 could not produce fumonisins.

\section{Focus on SM production with potential for industrial applications}

A LC-MS analysis was performed on a methanol extract obtained from 7-days culture of A. tubingensis G131 grown on CYA plate at $28{ }^{\circ} \mathrm{C}$ (Table 4). According to these data and those from Nielsen et al. [1] on biochemical compounds produced by $A$. niger clade, A. tubingensis G131 has the capacity to produce asperazine and 7
NGPs: fonsecin (TMC-256B1), rubrofusarin, aurasperones B, C, D, E and F. Therefore, a genomic search was carried out to identify the putative SM clusters involved in asperazine and NGPs biosynthesis.

Putative SM cluster for asperazine biosynthesis Asperazine, a diketopiperazine of the ditryptophenaline family (Fig. 4a), is a complex dimer alkaloid. It was first isolated from a marine-derived $A$. tubingensis, initially reported as $A$. niger by Varogulu et al. [23]. According to Nielsen et al. [1], this compound is a valuable chemical marker allowing to distinguish $A$. tubingensis from $A$. niger. Currently, asperazine can be synthetically produced $[46,47]$. The elucidation of synthetic steps gives crucial indication/information about required enzymatic functions for its in vivo production and makes it easier to trace genes encoding biosynthetic enzymes. Moreover, some biosynthetic steps for production of other diketopiperazines are already known. For example, the biosynthetic gene cluster for ditryptophenaline production in A. flavus is described [48]. It is composed of three genes coding for a NRPS $(d t p A)$, a methyltransferase $(d p t B)$ and a cytochrome $\mathrm{P} 450(\mathrm{dptC})$. It was also demonstrated that production of fumitremorgin in A. fumigatus required 3 cytochromes $\mathrm{P} 450$ ( $\mathrm{ftmE}, \mathrm{ftmC}$ and $\mathrm{ftm} G$ ) for cyclization, hydroxylation of the indole ring and hydroxylation of fumitremorgin $C$, respectively [49]. Among the $80 \mathrm{SM}$ clusters predicted in the A. tubingensis G131 genome, only one contains genes coding for a NRPS, a methyltransferase and a cytochrome P450: cluster 46 which we consider potentially involved in asperazine production. According to the SM clusters classification established here, cluster 46 belongs to the "highly conserved in A. tubingensis strains" category (Table 3). Indeed, this cluster is poorly conserved in A. niger CBS 513.88 (cov: 53\%; id: 85\%) and A. kawachii IFO 4308 (cov: 68\%; id: 92\%). This is in agreement with the conclusion of Nielsen et al. proposing asperazine as a marker for A. tubingensis strains classification [1].

Table 4 LC-MS analysis of a methanol extract obtained from 7-days culture of A. tubingensis G131 on CYA plate at $28{ }^{\circ} \mathrm{C}$. RT: Retention Time; MM: Monoisotopic Mass

\begin{tabular}{|c|c|c|c|c|c|c|c|}
\hline \multicolumn{4}{|c|}{ LC-MS analysis (this study) } & \multicolumn{2}{|c|}{ secondary metabolites of A. niger clade (Nielsen et al. 2009) } & \multirow{2}{*}{$\begin{array}{l}\text { Elementary } \\
\text { composition }\end{array}$} & \multirow[t]{2}{*}{ Secondary Metabolite } \\
\hline $\mathrm{RT}$ (min) & {$[\mathrm{M}-\mathrm{H}]^{+}$} & {$[\mathrm{M}-\mathrm{H}]^{-}$} & UVNis & $\mathrm{MM}(\mathrm{Da})$ & UVNis & & \\
\hline 17 & 291 & 289 & $230 ; 276 ; 333 ; 405$ & 290.08 & & $\mathrm{C}_{15} \mathrm{H}_{14} \mathrm{O}_{6}$ & Fonsecin (TMC-256B1) \\
\hline 25 & 665 & 663 & $225 ; 275 ; 300$ & 664.28 & $225 ; 275 ; 300$ & $\mathrm{C}_{40} \mathrm{H}_{36} \mathrm{~N}_{6} \mathrm{O}_{4}$ & Asperazine \\
\hline 27.5 & 272 & 271 & $223 ; 282 ; 333 ; 405$ & 272.07 & $225 ; 278 ; 328 ; 415$ & $\mathrm{C}_{15} \mathrm{H}_{12} \mathrm{O}_{5}$ & Rubrofusarin \\
\hline 31 & 593 & 592 & $234 ; 282 ; 334 ; 405$ & 592.16 & $236 ; 283.5 ; 323 ; 334 ; 412$ & $\mathrm{C}_{31} \mathrm{H}_{28} \mathrm{O}_{12}$ & Aurasperone $\mathrm{C}$ \\
\hline 33 & 575 & 573 & $228 ; 281 ; 321 ; 334$ & 574.15 & $213 ; 281 ; 320 ; 334$ & $\mathrm{C}_{31} \mathrm{H}_{26} \mathrm{O}_{11}$ & Aurasperone F \\
\hline 35 & 607 & 605 & $232 ; 282 ; 330 ; 405$ & 606.16 & $235 ; 282 ; 321 ; 334 ; 410$ & $\mathrm{C}_{32} \mathrm{H}_{30} \mathrm{O}_{12}$ & Aurasperone B \\
\hline \multirow[t]{2}{*}{37} & 589 & 587 & $224 ; 280 ; 407$ & 588.13 & $213 ; 281 ; 315 ; 332 ; 403$ & $\mathrm{C}_{32} \mathrm{H}_{28} \mathrm{O}_{11}$ & Aurasperone $\mathrm{E}$ \\
\hline & 557 & 555 & & 556.14 & $235-240 ; 280 ; 320-325 ; 380$ & $\mathrm{C}_{31} \mathrm{H}_{24} \mathrm{O}_{10}$ & Aurasperone D \\
\hline
\end{tabular}



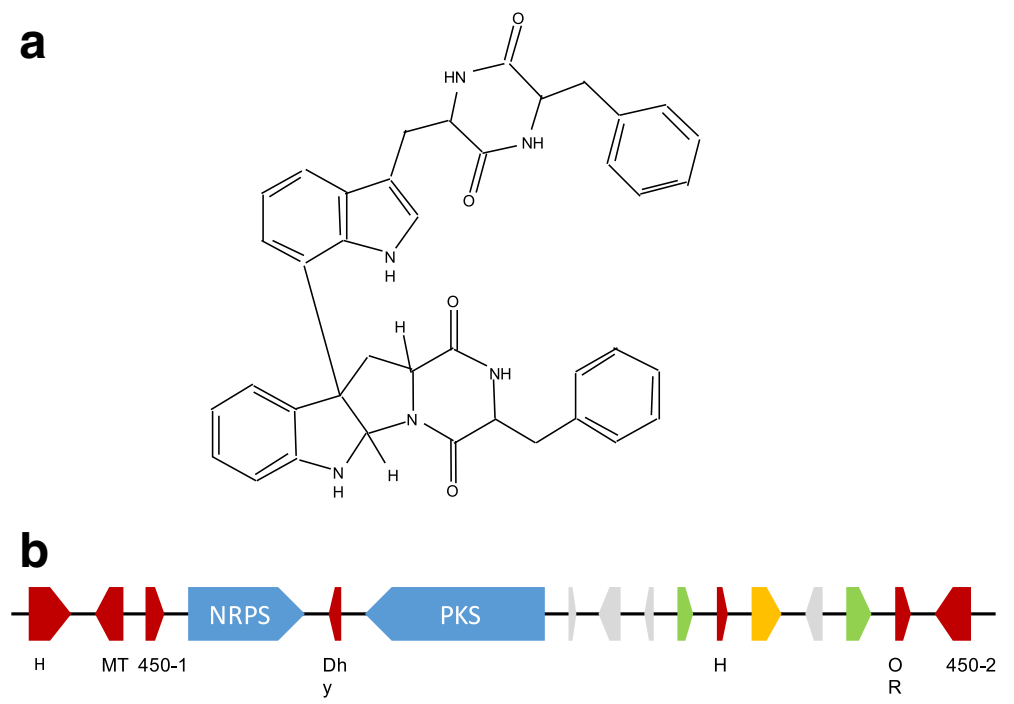

Fig. 4 Asperazine biosynthesis in A. tubingensis G131 - a. Asperazine structure b. Schematic representation of the putative asperazine SM cluster (cluster 46). Arrows indicate putative genes in the cluster and its direction indicates forward and reverse strand. Blue: gene coding for a backbone enzyme, Red: gene coding for an enzyme with known function, Green: gene coding for a transcription factor, Yellow: gene coding for a transporter, Grey: gene coding for an hypothetical protein. Below each biosynthetic gene, the putative function is indicated. Dhy: dehydratase, H: hydrolase, OR: oxidoreductase, MT: methyltransferase, 450: cytochrome P450

As shown Fig. 4b, SM cluster 46 is composed with genes coding for 2 transcription factors, 1 transporter, 2 backbone enzymes (1 PKS and 1 NRPS), a methyltransferase and 2 cytochromes P450. It also contains genes coding for enzymatic functions such as hydrolase, dehydratase or oxidoreductase activities. The conserved domains of the putative NRPS, PKS, methyltransferase and the 2 P450 cytochromes are available in Additional file 1: Figure S10.

Blast analyses of Cluster 46 were performed with all data available on diketopiperazines biosynthesis. This cluster shows homology with the fumitremorgin biosynthesis cluster identified in A. fumigatus Af293 (cov: 44\%; id: 89\%, Additional file 1: Table S5). Moreover, NRPS and methyltransferase of Cluster 46 show homology with DptA and DptB of A. flavus respectively (Additional file 1: Table S5). However, none of the two cytochromes P450 present in the SM cluster 46 shows homology with DptC. Indeed, asperazine dimerization is based on a C3-C7 linkage which is not observed in such compounds so far [50]. Kishimoto et al. proposed that biosynthesis of dimeric diketopiperazines, such as asperazine, is catalyzed by $\mathrm{P} 450$ cytochromes with relaxed substrate specificity [50]. This suggest that cytochromes $\mathrm{P} 450$ putatively required for asperazine production could be different from the one of ditryptophenaline biosynthesis in A. flavus and that cluster 46 could be the SM cluster for asperazine biosynthesis in A. tubingensis G131. However, to confirm the involvement of cluster 46 in asperazine synthesis, genetic experiments should be performed.
Putative SM cluster for NGPs biosynthesis As already described, fungi from the $A$. niger clade are NGPs producers [1]. Depending of the species, produced NGP dimers can vary: aurasperones, asperspyrones, nigerasperones or fonsecinones [2]. LC-MS analysis of a methanol extract indicates that A. tubingensis G131 is able to produce the NGPs monomers fonsecin and rubrofusarin and NGPs dimers members of aurasperones (Fig. 5a). These secondary metabolites are synthesized through cyclization of acetyl-CoA and malonylCoA precursors by a PKS identified as AlbA / PksP on $A$. niger ATCC 11414 and $A$. niger $\mathrm{N} 402$ [51, 52]. This PKS known as the "yellow conidial pigmentation PKS" is now well characterized in diverse fungi, especially in Aspergillus spp.. It is described that this PKS is involved in the formation of YWA1, which is a common precursor of both NGPs and DHN-melanin pigments [52]. After this step, little is known about NGPs biosynthesis [2].

To identify the SM cluster that could be involved in NGPs biosynthesis in A tubingensis G131, a BlastP analysis was performed to find the putative A. tubingensis G131 AlbA / PksP. Two candidates were selected: PKS of SM cluster 54 and PKS of SM cluster 16 (Distance tree results of the two predicted PKS putatively involved in NGPs synthesis are available in Additional file 1: Figure S11). According to the SM clusters classification established here, both clusters belong to the "highly conserved in A. tubingensis, A. niger, A. kawachii strains" category (Table 3 ), which corresponds to the assumption that all strains from the $A$. niger clade are able to produce NGPs. 


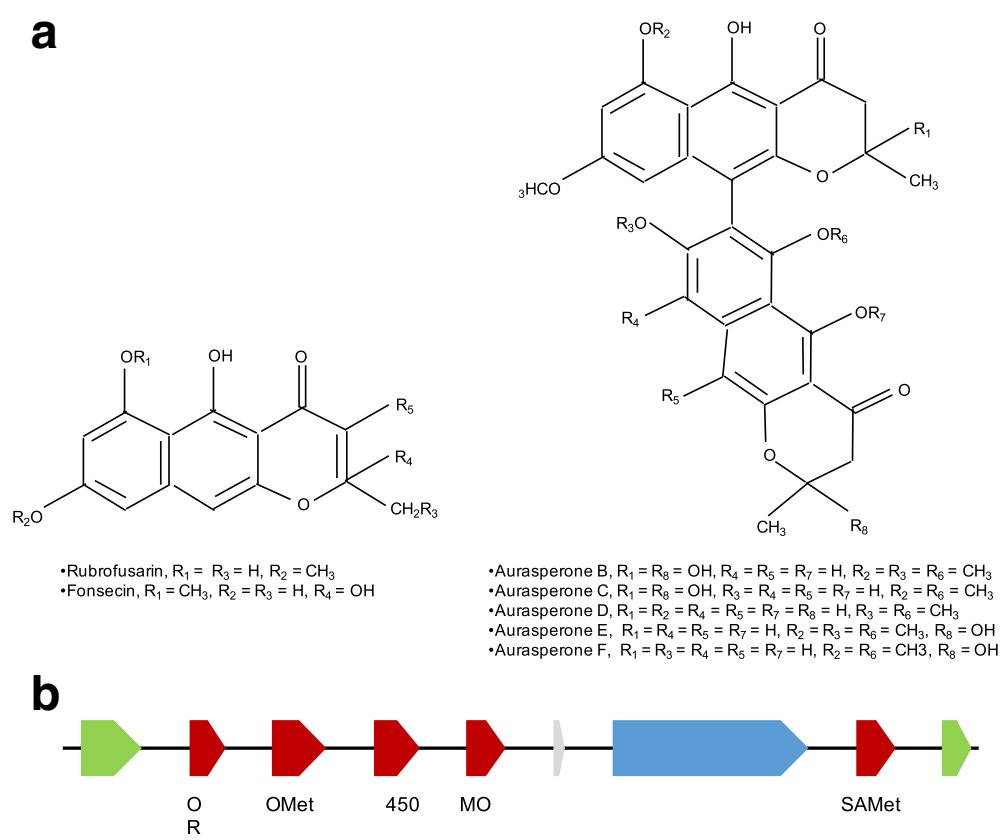

Fig. 5 NGPs biosynthesis in A. tubingensis G131 - a. Schematic representation of the putative NGP SM Cluster (Cluster 16). The representation was manually designed with information obtained from cluster prediction (AntiSMASH, SMURF), genes prediction (Augustus) and Annotation (AntiSMASH) b. Schematic representation of the putative NGP SM cluster (cluster 16). Arrows indicate putative genes in the cluster and its direction indicates forward and reverse strand. Blue: gene coding for a backbone enzyme, Red: gene coding for an enzyme with known function, Green: gene coding for a transcription factor, Grey: gene coding for an hypothetical protein. Below each biosynthetic gene, the putative function is indicated. SAMet: Sadenosylmethionine-dependent methyltransferase; OMet: O-methyltransferase; MO: monooxygenase; OR: oxidoreductase; 450: cytochrome P450

Distance trees show that the predicted PKS from cluster 54 has a strongest homology to AlbA / PksP (id: 96\%) than the predicted PKS from cluster 16 (id: 87\%). Moreover, genomic sequence of cluster 54 shows homology with the genomic region of $A$. tubingensis CBS 134.48 coding for SM cluster Asptu1.62 (JGI information). This putative SM cluster corresponds to a unique PKS, which is non-clusterized in A. tubingensis CBS 134.48. Comparing AntiSMASH and SMURF prediction for cluster 54 in A. tubingensis G131, a mismatch can be observed. SMURF identifies this PKS as a non-clusterized PKS, validating the result previously obtained on A. tubingensis CBS 134.48 [20]. Those results are in agreement with results previously obtained in different $A$. niger strains showing that the PKS involved in biosynthesis of both NGPs and DHN-melanin is non-clusterized $[2,51,52]$. For this reason, we suggest that cluster 54 PKS is homologue to AlbA / PksP and most likely the entire SM cluster is not solely involved in NGPs biosynthesis.

PKS from cluster 54 and 16 are orthologues and share the same conserved domains organization: SAT-KS-ATACP-ACP-TE (Additional file 1: Figures S11 \& S12). The same conserved domains organization was observed for two PKS in A. niger ATCC 1015: AlbA and PKS44 [16]. According to this result, we suggest that cluster 16 PKS could lead to the same type of cyclization than AlbA / $\mathrm{PksP}$ and be involved in the synthesis of the same type of intermediate. Looking further into functions of cluster 16 predicted proteins (Fig. $5 \mathrm{~b}$ ), it appears that genes of this cluster encode for 2 transcriptional factors, 1 Cytochrome P450, 1 monooxygenase, 2 methyltransferases, 1 oxidoreductase and 1 protein of unknown function. According to the structure of NGPs produced by A. tubingensis G131, after PKS cyclization, the main enzyme activities required for their synthesis are hydroxylation, o-methylation and dimerization. Hydroxylation is catalyzed by monooxygenases, o-methylation by omethyltransferases and some cytochromes $\mathrm{P} 450$ or other types of oxidoreductases could catalyze dimerization. Cluster 16 possesses all the required functions for the biosynthesis of NGPs. This is not the case of cluster 54, which lacks oxidoreductases. Moreover, among the two transcription factors identified in cluster 16 , one is a zinc finger transcriptional factor which could be involved in the regulation of the entire cluster, and the other is a NmrA-type transcriptional factor, specific to oxidoreductase negative regulation which could be involved in dimerization control. For these reasons, we hypothesize that cluster 16 could be the putative SM cluster for the biosynthesis of NGPs in A. tubingensis G131. However, this hypothesis needs to be validated by genetic analyses.

The variability of equivalent SM clusters through the A. niger clade (Genetic distance and genes similarity of 
Aspergillus sp. with cluster16 are available in Additional file 1: Figure S13) could be explained by the different NGP types produced by the strains, and conserved domains required for their synthesis. However, a deeper comparative genomic and biochemical analysis should be performed to link the differences between cluster sequences and the putative NGPs produced.

\section{Conclusions}

An increasing amount of knowledge about black Aspergilli is becoming available, largely derived from genome sequencing projects. In this study, we report the genome draft of A. tubingensis G131 and highlight its secondary metabolism potential. Indeed, $80 \mathrm{SM}$ biosynthetic gene clusters were identified in the genome assembly. Comparisons of these SM clusters with genome assemblies of A. tubingensis CBS 134.48, A. niger CBS 513.88 and A. kawachii IFO 4308 allow identification of seven clusters described for the first time and putatively unique. However, sequencing of other $A$. tubingensis strains, future comparative genomic analyses and biochemical and genetic characterization will be necessary to validate and confirm the singularity of these putative SM clusters.

Genes coding enzymes involved in OTA and fumonisins synthesis in black Aspergilli were systematically searched. This genomic analysis allows to confirm biochemical results previously obtained by Bouras et al. [25] and showing that $A$. tubingensis G131 is not able to produce these two types of mycotoxins. Besides, the genomic analyses performed here suggest that $A$. tubingensis G131, isolated from a French Mediterranean vineyard, is a good candidate to produce natural compounds with interesting biological properties such as asperazine (antibiotic) and NGPs (antioxidant, anticancer, antibiotic). Genomic studies also suggest that the strain can produce anthracenone / naphthacenedione with putative immunosuppressive properties $[40,46]$.

\section{Methods}

\section{Culture and genomic DNA extraction}

A. tubingensis G131 was isolated from a French Mediterranean vineyard, classified into Aspergillus section Nigri (morphological characterization) and described as nonochratoxigenic (biochemical characterization) [25]. The strain was cultured 3 days at $28^{\circ} \mathrm{C}$ under shaking condition $120 \mathrm{rpm}$ on PDB medium. The fungal mycelial mat was harvested and ground into a fine powder with liquid nitrogen and conserved at $-80{ }^{\circ} \mathrm{C}$ until used. Genomic DNA was extracted from this powder with a protocol adapted from [53]. $150 \mathrm{mg}$ of mycelial powder was transferred to a pre-cooled Eppendorf tube with $700 \mu \mathrm{l}$ of CTAB solution (1\% CTAB; $100 \mathrm{mM} \mathrm{NaCl} ; 100 \mathrm{mM}$ EDTA pH 8; $20 \mathrm{mM}$ Tris- $\mathrm{Cl} \mathrm{pH}$ 8), homogenized by vortex and incubated $1 \mathrm{~h}$ on ice. Genomic DNA was then extracted using two successive Phenol/Chloroform/ Isoamyl Alcohol (Sigma) washes, precipitated with anhydrous ethanol and suspended in nuclease free water. Genomic DNA concentration and quality were estimated using Nanodrop 2000 (Eppendorf).

\section{Genome sequencing and assembly}

The Illumina MiSeq platform was used for the wholegenome shotgun sequencing of $A$. tubingensis G131. Pairends sequences of $2 * 300 \mathrm{bp}$ were produced. The raw sequence data were trimmed with trim_galore v0.4.0 [54] and a quality control was performed with fastqc [55]. Genome assembly was performed with discovardenovo-52,488 [56]. Statistics of this assembly were collected using the assemblathon_stats.pl script [57].

\section{Genome annotation and taxonomy Genome annotation}

Genome annotation was done using Augustus gene prediction software [32] using $A$. nidulans as a reference. Then, predicted proteins were annotated by homology search using TblastN on NCBI and BlastX on Interproscan databases through Blast2GO software (default parameters) [33]. Blast2GO results allow comparing annotation in the Gene Onthology databases to identify putative molecular functions and cellular processes acquired by the strain.

\section{Taxonomy}

Taxonomy was performed through molecular identification by alignment of reference genes RNA Polymerase Subunit 2 (RPB2), beta-tubulin (BenA) and Calmodulin (Cam1) as described in $[29,58,59]$. DNA sequences were BlastN on NCBI database and then aligned using Clustal omega algorithm [60]. Each locus was aligned separately and then concatenated in a super-gene alignment, as described in [29] used to generate the phylogenetic tree, with clustal phylogeny [60] based on Neighbor-Joining method. Bootstrap values were computed from 100 replications of the bootstrap procedure using phylogeny.fr and added to the phylogenetic tree [61]. All positions containing gaps and missing data were eliminated from the dataset (complete deletion option).

\section{Genome comparative analyses \\ Comparative wide comparison of orthologous clusters}

Genome wide analysis of orthologous clusters was done through the interactive plateform OrthoVenn [36] using A. tubingensis G131, A. tubingensis CBS 134.48 [20], A. niger CBS 513.88 [13] and A. kawachii IFO 4308 [21] predicted proteomes. 
R2Cat

Syntheny analyses between $A$. tubingensis G131 and $A$. tubingensis CBS 134.48 were addressed with r2cat software [62].

\section{KOG analyses}

Clusters of Orthologous Groups of proteins (COGs) from the sequenced genomes of $A$. tubingensis G131, $A$. tubingensis CBS 134.48 [20], A. niger CBS 513.88 [13] and $A$. kawachii IFO 4308 [21] were analysed using the COG database [35].

\section{Secretome analysis}

The predictive secretome was obtained using SignalP with default parameters [63]. Each genome analysis was addressed independently.

\section{Identification of secondary metabolites biosynthetic gene clusters}

The identification of SM biosynthetic gene clusters was performed both with SMURF software [38] and AntiSMASH [39]. SMURF is based on cluster search on the annotated proteome and found putative clusters through conserved domain homology. On the contrary AntiSMASH works directly on the genome scaffolds assembly and find biosynthetic gene clusters through homology with known clusters. The clusters obtained with both methodologies were compared through blast analyses and concatenated for the final SM biosynthetic gene cluster prediction. Concatenation is based on the choice of SM cluster boundaries, to have the longest clusters.

\section{Culture and secondary metabolites profiling Culture}

A. tubingensis $\mathrm{G} 131$ was cultured at $28{ }^{\circ} \mathrm{C}$, on CYA plates (CYA - $30 \mathrm{~g} / \mathrm{L}$ Saccharose, $15 \mathrm{~g} / \mathrm{L}$ agar, $5 \mathrm{~g} / \mathrm{L}$ Yeast Extract, $2 \mathrm{~g} / \mathrm{L} \mathrm{NaNO}_{3}, 0.25 \mathrm{~g} / \mathrm{L} \mathrm{KCl}, 0.25 \mathrm{~g} / \mathrm{L}$ $\mathrm{MgSO}_{4} .7 \mathrm{H}_{2} \mathrm{O}, 0.005 \mathrm{~g} / \mathrm{L} \mathrm{FeSO}_{4} .7 \mathrm{H}_{2} \mathrm{O}, 0.5 \mathrm{~g} / \mathrm{L} \mathrm{K}_{2} \mathrm{HPO}_{4}$, $0.001 \mathrm{~g} / \mathrm{L} \mathrm{ZnSO}_{4} .7 \mathrm{H}_{2} \mathrm{O}$ and $\left.0.0005 \mathrm{~g} / \mathrm{L} \mathrm{CuSO}_{4} \cdot 7 \mathrm{H}_{2} \mathrm{O}\right)$. After 7 days, the agar plate containing mycelium and spores was covered with methanol. The mycelium / methanol mix was incubated $20 \mathrm{~min}$ at room temperature and sonicated at $50 \mathrm{~Hz}$ for additional $20 \mathrm{~min}$. After sonication, the mycelium / methanol mix was filtered once on $113 \mathrm{~V}$ grade Whatman filter paper. The obtained filtered extract was conserved at $4{ }^{\circ} \mathrm{C}$ in the dark until HPLC analysis.

\section{LC-MS analysis}

HPLC were run on Ultimate 3000 DIONEX device, equipped of a Prontosil 120-5-C18 column (150× $4.6 \mathrm{~mm}, \mathrm{SH}$ : $50 \mu \mathrm{m})$. Samples were conditioned in $1 \mathrm{ml}$ untainted glass vials, filtered with PTFE $0.45 \mu \mathrm{m}$ filter.
$10 \mu \mathrm{L}$ was injected for analysis. Acquisition was done between 200 and $600 \mathrm{~nm}$ while column was maintained at $30{ }^{\circ} \mathrm{C}$. Compounds separation was obtained by a 40 min linear gradient from acetonitrile-water-acetic acid (30: 69.9: 0.1, v/v) to pure acetonitrile, followed by a 5 min isocratic step of pure acetonitrile and then equilibrated during $5 \mathrm{~min}$ to acetonitrile-water-acetic acid (30, 69.9: $0.1, \mathrm{v} / \mathrm{v}$ ), at a flow rate of $0.8 \mathrm{~mL} / \mathrm{min}$.

Mass spectrometry analyses were realized on a MS/ MS Q-TRAP system (Applied Biosystems ${ }^{\oplus}$ ) with a ESI as ionization source. Analyses were runned in both positive and negative modes.

\section{Additional file}

Additional file 1: Table S1. BUSCO analysis of A. tubingensis G131 scaffolds assembly. Figure S1. Dot Plot analysis between A. tubingensis G131 and A. tubingensis CBS 148.33 to order the scaffold assembly. Table S2. Accession number (NCBI) of sequences used for phylogenetic analysis. Figure S2. Phylogenetic tree produced from Rpb2 partial gene sequence of 38 strains of black aspergilli. Figure S3. Phylogenetic tree produced from Cam 1 partial gene sequence of 38 strains of black aspergilli. Figure S4. Phylogenetic tree produced from BenA partial gene sequence of 38 strains of black aspergilli. Figure S5. Blast2GO statistics summary. Table S3. KOG analysis of $A$. tubingensis G131, A. tubingensis CBS 134.48, A. kawachii IFO 4308 and A. niger CBS 513.88. Figure S6. Venn Diagram of secretome analysis obtained through SignalP and OrthoMCL analysis. Table S4. SM Clusters coordinates of the scaffolds assembly. Figure S7. Genes similarity between SM cluster 44 from A. tubingensis G131 and others known fungal biosynthetic cluster. (Results obtained with AntiSMASH software). Figure S8. AntiSMASH schematic representation of the conserved domains in SM clusters unique in $A$. tubingensis G131 and their similarities with other SM clusters identified in various fungi (AntiSMASH results). Table S5. Blast results for Ochratoxin A, Fumonisins and asperazine biosynthesis in A. tubingensis. Figure S9. Schematic representation of Cluster 4 presenting small homology with fumonisin cluster. Figure S10. Repartition of conserved domains in predictive enzymes coding genes of the asperazine cluster obtained with CD search. Figure S11. Conserved domain (CD-Search) and Distance tree results obtained from NCBI BlastP analysis of predicted PKS of $A$. tubingensis G131 putatively involved in NGPs synthesis. Figure S12. Clustal Omega Alignment of predicted PKS from SM Clusters 54 and 16 of $A$. tubingensis G131. Figure S13. Genes similarity between Cluster 16 from $A$. tubingensis $\mathrm{G} 131$ with other known fungal genomes assembly. (PDF $2680 \mathrm{~kb}$ )

\section{Abbreviations}

COGs: Clusters of Orthologous Groups of proteins; Cov: coverage; CYA: Czapech Yeast Agar; Id: identity; NGPs: Naphtho-Gamma-Pyrones; NRPS: Non-ribosomal peptide synthase; OTA: Ochratoxin A; PKS: Polyketide Synthase; SM: Secondary Metabolite; TC: Terpene cyclase; WGS: Whole Genome Shotgun

\section{Acknowledgements}

Not applicable.

Funding

Not applicable.

\section{Availability of data and materials}

The genome scaffolds assembly of A. tubingensis G131 generated and analyzed during this study has been deposited at European Nucleotide Archive (ENA) under the accession numbers FUKH01000001 - FUKH01000192. Phylogenic analysis presented in Fig. 2 is available on TreeBase at the following URL: http://purl.org/phylo/treebase/phylows/study/TB2:S22371 


\section{Authors' contributions}

EC performed genomic DNA extraction, bioinformatic analyses and wrote the main manuscript text, SV performed the sequencing, CK performed genome assembly and bioinformatic analyses. JR and FM made a critical revision of the manuscript. All authors read and approved the final manuscript.

\section{Ethics approval and consent to participate}

No permission was needed to collect the samples used in this study.

\section{Consent for publication}

Not applicable.

\section{Competing interests}

The authors declare that they have no competing interests.

\section{Publisher's Note}

Springer Nature remains neutral with regard to jurisdictional claims in published maps and institutional affiliations.

\section{Author details}

'Université de Toulouse, Laboratoire de Génie Chimique, UMR 5503 CNRS/ INPT/UPS, INP-ENSAT, 1, avenue de I'Agrobiopôle, 31326 Castanet-Tolosan, France. ${ }^{2}$ Present address: Unité de Recherche Biologie des Plantes et Innovation (BIOPI-EA 3900), Université de Picardie Jules Verne, 33 rue Saint Leu, 80039 Amiens Cedex, France. ${ }^{3}$ Plate-forme Genotoul Bioinfo, UR875 Biométrie et Intelligence Artificielle, Institut National de la Recherche Agronomique, Castanet-Tolosan, France. ${ }^{4}$ INRA, US 1426, GeT-PlaGe, Genotoul, Castanet-Tolosan, France.

\section{Received: 25 October 2017 Accepted: 2 March 2018}

\section{Published online: 15 March 2018}

\section{References}

1. Nielsen KF, Mogensen JM, Johansen M, Larsen TO, Frisvad JC. Review of secondary metabolites and mycotoxins from the Aspergillus niger group. Anal Bioanal Chem. 2009:395(5):1225-42.

2. Choque E, El Rayess Y, Raynal J, Mathieu F. Fungal naphtho- $\gamma$-pyronessecondary metabolites of industrial interest. Appl Microbiol Biotechnol. 2015;99(3):1081-96.

3. Ferreira JA, Mahboubi A, Lennartsson PR, Taherzadeh MJ. Waste biorefineries using filamentous ascomycetes fungi: present status and future prospects. Bioresour Technol. 2016;215:334-45.

4. Mulder KCL, Mulinari F, Franco OL, Soares MSF, Magalhães BS, Parachin NS Lovastatin production: from molecular basis to industrial process optimization. Biotechnol Adv. 2015:33(6):648-65.

5. Lamboni $Y$, et al. Diversity in secondary metabolites including mycotoxins from strains of Aspergillus section Nigri isolated from raw cashew nuts from Benin, West Africa. PLoS One. 2016; https://doi.org/10.1371/journal.pone.0164310.

6. Cho SM, et al. Biodegradation of Ochratoxin a by Aspergillus tubingensis isolated from Meju. J Microbiol Biotechnol. 2016;26(10):1687-95.

7. Qi TF, Renaud JB, McDowell T, Seifert KA, Yeung KK-C, Sumarah MW. Diversity of mycotoxin-producing black aspergilli in Canadian vineyards. J Agric Food Chem. 2016;64(7):1583-9.

8. Blumenthal CZ. Production of toxic metabolites in Aspergillus niger, Aspergillus oryzae, and Trichoderma reesei: justification of mycotoxin testing in food grade enzyme preparations derived from the three fungi. Regul Toxicol Pharmacol. 2004;39(2):214-28.

9. Schuster E, Dunn-Coleman N, Frisvad J, van Dijck P. On the safety of Aspergillus niger - a review. Appl Microbiol Biotechnol. 2002;59(4-5):426-35.

10. Wang Q, Gong J, Chisti Y, Sirisansaneeyakul S. Production of theabrownins using a crude fungal enzyme concentrate. J Biotechnol. 2016;231:250-9.

11. Dos Santos JA, et al. Marine-derived fungus Aspergillus cf. tubingensis LAMAl 31 : a new genetic resource for xylanase production. AMB Express. 2016;6(1):25.

12. Trivedi S, Divecha J, Shah A. Optimization of inulinase production by a newly isolated Aspergillus tubingensis CR16 using low cost substrates. Carbohydr Polym. 2012;90(1):483-90.

13. Pel HJ, et al. Genome sequencing and analysis of the versatile cell factory Aspergillus niger CBS 513.88. Nat Biotechnol. 2007;25(2):221-31.

14. Andersen MR, et al. Comparative genomics of citric-acid-producing Aspergillus niger ATCC 1015 versus enzyme-producing CBS 513.88. Genome Res. 2011;21(6):885-97.
15. Inglis DO, et al. Comprehensive annotation of secondary metabolite biosynthetic genes and gene clusters of Aspergillus nidulans, A. fumigatus, $A$ niger and A. oryzae. BMC Microbiol. 2013;13:91.

16. Fisch KM, et al. Chemical induction of silent biosynthetic pathway transcription in Aspergillus niger. J Ind Microbiol Biotechnol. 2009;36(9): 1199-213.

17. Sanchez JF, Somoza AD, Keller NP, Wang CCC. Advances in Aspergillus secondary metabolite research in the post-genomic era. Nat Prod Rep. 2012;29(3):351-71.

18. Cabañes FJ, Sanseverino W, Castellá G, Bragulat MR, Cigliano RA, Sánchez A. Rapid genome resequencing of an atoxigenic strain of Aspergillus carbonarius. Sci Rep. 2015;5:9086

19. Sartori D, Massi FP, Ferranti LS, Fungaro MHP. Identification of genes differentially expressed between Ochratoxin-producing and non-producing strains of aspergillus westerdijkiae. Indian J Microbiol. 2014;54:41-5.

20. de Vries RP, et al. Comparative genomics reveals high biological diversity and specific adaptations in the industrially and medically important fungal genus Aspergillus. Genome Biol. 2017; https://doi.org/10.1186/s13059-017-1151-0.

21. Futagami T, et al. Genome sequence of the white koji mold Aspergillus kawachii IFO 4308, used for brewing the Japanese distilled spirit shochu. Eukaryot Cell. 2011:10(11):1586-7.

22. Frisvad JC, Smedsgaard J, Samson RA, Larsen TO, Thrane U. Fumonisin B2 production by Aspergillus niger. J Agric Food Chem. 2007;55(23):9727-32.

23. Varoglu M, Corbett TH, Valeriote FA, Crews P. Asperazine, a selective cytotoxic alkaloid from a sponge-derived culture of Aspergillus niger. J Org Chem. 1997;62(21):7078-9.

24. Li X-B, Li Y-L, Zhou J-C, Yuan H-Q, Wang X-N, Lou H-X. A new diketopiperazine heterodimer from an endophytic fungus Aspergillus niger. J Asian Nat Prod Res. 2015;17(2):182-7.

25. Bouras N, Mathieu F, Coppel Y, Strelkov SE, Lebrihi A. Occurrence of naphthogamma-pyrones- and ochratoxin A-producing fungi in French grapes and characterization of new naphtho-gamma-pyrone polyketide (aurasperone G) isolated from Aspergillus niger C-433. J Agric Food Chem. 2007;55(22):8920-7.

26. Simão FA, Waterhouse RM, loannidis P, Kriventseva EV, Zdobnov EM. BUSCO: assessing genome assembly and annotation completeness with single-copy orthologs. Bioinforma Oxf Engl. 2015;31(19):3210-2.

27. O. Yamada et al. Genome sequence of Aspergillus luchuensis NBRC 4314 DNA Res Int J Rapid Publ Rep Genes Genomes 2016. doi:https://doi.org/10. 1093/dnares/dsw032.

28. Goris J, Konstantinidis KT, Klappenbach JA, Coenye T, Vandamme P, Tiedje JM. DNA-DNA hybridization values and their relationship to whole-genome sequence similarities. Int J Syst Evol Microbiol. 2007;57:81-91.

29. Samson RA, Noonim P, Meijer M, Houbraken J, Frisvad JC, Varga J. Diagnostic tools to identify black aspergilli. Stud Mycol. 2007;59:129-45.

30. Jurjević Z, et al. Two novel species of Aspergillus section Nigri from indoor air. IMA Fungus. 2012;3(2):159-73.

31. Taylor JW, et al. Phylogenetic species recognition and species concepts in fungi. Fungal Genet Biol. 2000;31(1):21-32

32. Hoff KJ, Stanke M. WebAUGUSTUS-a web service for training AUGUSTUS and predicting genes in eukaryotes. Nucleic Acids Res. 2013; https://doi.org/ 10.1093/nar/gkt418.

33. Conesa A, Götz S, García-Gómez JM, Terol J, Talón M, Robles M. Blast2GO: a universal tool for annotation, visualization and analysis in functional genomics research. Bioinforma Oxf Engl. 2005;21(18):3674-6.

34. Wang D, Hsieh M, Li W-H, General Tendency A. For conservation of protein length across eukaryotic kingdoms. Mol Biol Evol. 2005;22(1):142-7.

35. Tatusov RL, et al. The COG database: an updated version includes eukaryotes. BMC Bioinformatics. 2003:4:41.

36. Wang Y, Coleman-Derr D, Chen G, Gu YQ. OrthoVenn: a web server for genome wide comparison and annotation of orthologous clusters across multiple species. Nucleic Acids Res. 2015;43(W1):78-84.

37. Lind $A L_{\text {, }}$ et al. Drivers of genetic diversity in secondary metabolic gene clusters within a fungal species. PLoS Biol. 2017;15(11):e2003583.

38. Khaldi N, et al. SMURF: genomic mapping of fungal secondary metabolite clusters. Fungal Genet Biol. 2010;47(9):736-41.

39. Weber $\mathrm{T}$, et al. antiSMASH 3.0-a comprehensive resource for the genome mining of biosynthetic gene clusters. Nucleic Acids Res. 2015;43:237-43.

40. Li Y, Chooi Y-H, Sheng Y, Valentine JS, Tang Y. Comparative characterization of fungal anthracenone and naphthacenedione biosynthetic pathways reveals an a-hydroxylation-dependent Claisen-like cyclization catalyzed by a dimanganese thioesterase. J Am Chem Soc. 2011;133(39):15773-85. 
41. Kodukula K, et al. BMS-192548, a tetracyclic binding inhibitor of neuropeptide Y receptors, from Aspergillus niger WB2346. I. Taxonomy, fermentation, isolation and biological activity. J Antibiot. 1995;48(10):1055-9.

42. Palencia ER, Hinton DM, Bacon CW. The black Aspergillus species of maize and peanuts and their potential for mycotoxin production. Toxins. 2010;2(4):399-416.

43. Susca A, et al. Variation in the fumonisin biosynthetic gene cluster in fumonisin-producing and nonproducing black aspergilli. Fungal Genet Biol. 2014;73:39-52

44. Massi FP, et al. Data on the presence or absence of genes encoding essential proteins for ochratoxin and fumonisin biosynthesis in Aspergillus niger and Aspergillus welwitschiae. Data Brief. 2016;7:704-8.

45. Storari M, Bigler L, Gessler C, Broggini GAL. Assessment of the ochratoxin a production ability of Aspergillus tubingensis. Food Addit Contam Part Chem Anal Control Expo Risk Assess. 2012;29(9):1450-4.

46. Govek SP, Overman LE. Total synthesis of (+)-Asperazine. J Am Chem Soc 2001;123(38):9468-9.

47. Loach RP, Fenton OS, Movassaghi M. Concise Total synthesis of (+)-Asperazine, (+)-Pestalazine a, and (+)-iso-Pestalazine a. Structure revision of (+)-Pestalazine a. J Am Chem Soc. 2016;138(3):1057-64.

48. Payne GA, et al. Whole genome comparison of Aspergillus flavus and A. oryzae. Med Mycol. 2006;44(S1):S9-S11.

49. Kato $\mathrm{N}$, et al. Identification of cytochrome P450s required for fumitremorgin biosynthesis in Aspergillus fumigatus. Chembiochem Eur. J Chem Biol. 2009; 10(5):920-8.

50. Kishimoto S, Sato M, Tsunematsu Y, Watanabe K. Evaluation of biosynthetic pathway and engineered biosynthesis of alkaloids. Mol Basel Switz. 2016; https://doi.org/10.3390/molecules21081078.

51. Jørgensen TR, et al. The molecular and genetic basis of conidial pigmentation in Aspergillus niger. Fungal Genet Biol. 2011;48(5):544-53.

52. Chiang Y-M, Meyer KM, Praseuth M, Baker SE, Bruno KS, Wang CCC.

Characterization of a polyketide synthase in Aspergillus niger whose product is a precursor for both dihydroxynaphthalene (DHN) melanin and naphthoy-pyrone. Fungal Genet Biol. 2011;48(4):430-7.

53. van Burik JA, Schreckhise RW, White TC, Bowden RA, Myerson D. Comparison of six extraction techniques for isolation of DNA from filamentous fungi. Med Mycol. 1998;36(5):299-303.

54. Trim galore v0.4.0. www.bioinformatics.babraham.ac.uk. Accessed: June 2015

55. fastqc. www.bioinformatics.babraham.ac.uk/projects/fastqc/. Accessed: June 2015.

56. Love RR, Weisenfeld NI, Jaffe DB, Besansky NJ, Neafsey DE. Evaluation of DISCOVAR de novo using a mosquito sample for cost-effective short-read genome assembly. BMC Genomics. 2016;17:187.

57. Bradnam KR, et al. Assemblathon 2: evaluating de novo methods of genome assembly in three vertebrate species. GigaScience. 2013;2:10.

58. Varga J, et al. New and revisited species in Aspergillus section Nigri. Stud Mycol. 2011;69(1):1-17.

59. Samson RA, et al. Phylogeny, identification and nomenclature of the genus Aspergillus. Stud Mycol. 2014;78:141-73.

60. Li W, et al. The EMBL-EBI bioinformatics web and programmatic tools framework. Nucleic Acids Res. 2015;43:580-4

61. Dereeper A, et al. Phylogeny.Fr: robust phylogenetic analysis for the nonspecialist. Nucleic Acids Res. 2008;36:465-9.

62. Husemann P, Stoye J. r2cat: synteny plots and comparative assembly. Bioinformatics. 2010;26(4):570-1.

63. Nielsen $\mathrm{H}$. Predicting secretory proteins with SignalP. Methods Mol Biol. 2017:1611:59-73.

\section{Submit your next manuscript to BioMed Central and we will help you at every step:}

- We accept pre-submission inquiries

- Our selector tool helps you to find the most relevant journal

- We provide round the clock customer support

- Convenient online submission

- Thorough peer review

- Inclusion in PubMed and all major indexing services

- Maximum visibility for your research

Submit your manuscript at www.biomedcentral.com/submit
Biomed Central 